\title{
New Metalized Polyimide Films Structure and Physical Properties
}

\author{
Saule K. Kudaikulova ${ }^{1,5}$, Rinat Iskakov ${ }^{1}$, Elena Vecherkina ${ }^{1,5}$, Bulat A. Zhubanov ${ }^{1}$, Oleg Prik- \\ hodko ${ }^{2}$, Andrey Kurbatov ${ }^{2}$, Tleuken Akhmetov ${ }^{2}$, Sergey Bazhenov ${ }^{3}$, Irina Razumovskaya ${ }^{3}$, Alain \\ Perichaud $^{4}$, Vanda Yu. Voytekunas ${ }^{5,6}$, Vitali Lipik ${ }^{5,7}$ and Marc J.M. Abadie*,5,7
}

\author{
${ }^{1}$ Institute of Chemical Sciences - ICS of Kazakh Academy of Science, 106 Valihanov, 480100 Alma-Ata, Republic of \\ Kazakhstan \\ ${ }^{2}$ el-Farabi Kazakh National University, 96 Karasay Batyr, 480012, Almaty, Republic of Kazakhstan \\ ${ }^{3}$ Russian State Pedagogical University, 2M. Pirogovskaya Street, Moscow, Russia \\ ${ }^{4}$ University of Provence Aix-Marseille, 3 Place Victor Hugo, 13331 Marseille, France \\ ${ }^{5}$ Laboratory of Polymer Science \& Advanced Organic Materials - LEMP/MAO, University Montpellier II, Place Batail- \\ lon, 34095 Montpellier Cedex 05, France \\ ${ }^{6}$ Singapore Institute of Manufacturing Technology - SIMTech, 71 Nanyang Drive, 638075, Singapore \\ ${ }^{7}$ School of Materials Science and Engineering, Nanyang Technological University - NTU, 50 Nanyang Avenue, 639798, \\ Singapore
}

\begin{abstract}
The classical techniques of PI film metallization by either vapour deposition or electrochemical reduction of metals involve necessary technological steps such as polymer surface modification via plasma or ion beam, electron beam, or photolytic treatment, or surface sensitization by the conferment of catalytic properties, in order to enhance metalPI adhesion, which is the main problem to be overcome in all processes.

The technology presented differs by the use of another chemical approach yielding electro-conductive materials with high reflectivity, but without damaging the films in the process. The metalized polyimide films have been prepared by heterogeneous chemical modification of the polyimide surface and the suggested technology includes several chemical conversion steps (hydrolysis, chelating by metal salts and reduction) requiring no complicated procedures and proceeding under mild conditions, at room temperature and without aggressive agents. This new process allows the formulation of metal phase while both simplifying the technology and making use of commercial PI films, such as Kapton ${ }^{\circledR} \mathrm{HN}$ and Upilex ${ }^{\circledR} \mathrm{S}$.
\end{abstract}

\section{In memoriam of Dr. Saule K. Kudaikulova}

\section{INTRODUCTION}

Metalized thin-film constructs [1-3] are one of the current objectives of space materials research towards developing new reflective materials. Such devices have wide application as thermo- regulative coatings, light reflectors, collectors of solar energy, antennas and also in the screen-vacuumthermoisolation. Easiness, good plastic and elastic properties of the polymer materials as well as the possibility of unfolding the constructions in space largely determine the prospects of their application. Pattern-like metallization techniques make thermostable polymeric substrates essential materials for microelectronic applications.

Such films are based on polyimides, which are characterized by high thermoplastic and radiate stability, and are in the most parts applied as polymer matrices. At present the aluminized film blends Kapton ${ }^{\mathbb{B}}$ (Du Pont, USA) are used in

*Address correspondence to this author at the School of Materials Science and Engineering, Nanyang Technological University - NTU, 50 Nanyang Avenue, 639798, Singapore; E-mail: marc@ntu.edu.sg

Laboratory of Polymer Science \& Advanced Organic Materials LEMP/MAO, University Montpellier II, Place Bataillon, 34095 Montpellier Cedex 05, France; E-mail: abadie@univ-montp2.fr space systems in low-earth orbits. The aluminized coating is stable towards the open space environment and resistant to atomic oxygen in particular. Due to their high reflectivity in a wide spectral range, the silvered polyimide films are the best prospective candidates for developing satellite antennas and telescopes for the transmission of information in IRspectral range in geostationary orbits where there is no factor of atomic oxygen.

There are two principles in the formation of reflective mirror surfaces: the secondary - precipitation of the metal on an optically transparent substrate layer, and the primary creation of the metal layer with the glossy surface. Because of the lack of the adhesion of silver to polyimide and the lack of optical transparency of polyimide films based on aromatic dianhydrides, the formation of the secondary mirror silver surfaces on polyimides is very difficult. In order to increase silver adhesion to polyimide surfaces, it is necessary to use special technological steps for morphological surface modification such as plasmochemical etching, ion - and electrobeam bombardment, or photolytic treatment. However, the silver adhesion does not increase strongly after these modification methods. 
To reduce the price of the production of these materials, and to use them more widely not only in space but also in the energy, electrotechnological and microelectronics fields, it is necessary to solve the problem of binding the primary mirror surface coating of high conductivity silver layers to the polyimide films. At present the main metallization methods include homogenous addition of metal compounds (salts or coordinating complexes) into the prepolymer solution with subsequent film casting [4-7] or the superfluid [8] impregnation of metal compounds into polyimide films. The modified films are then treated at high temperature (5-7 hours at no lower than $300{ }^{\circ} \mathrm{C}$ ). Along with thermal reduction in the course of film annealing, there is diffusion of metals to the film surface. But this does not create stable homogenous electroconductive metal coatings with high reflectivities. Other operations to optimize the process do not result in increased reflectivity and conductivity. It is to be noted that these methods do not use the standard industrially produced homopolyimide films for metallization. The present work is devoted to developing silvered polyimide films, investigation of their structure, metal distribution and electro-optical properties.

\section{EXPERIMENTAL}

Metalized polyimide films were prepared both in the Laboratory of the Polymer Synthesis at the Institute of Chemical Sciences (ICS, Almaty) and in the Laboratory of Polymer Science \& Advanced Organic Materials (LEMP/ MAO, Montpellier) by the method of chemical modification. The silver coatings on the substrate were obtained by the precipitation of silver by chemical reduction of silver nitrate with Li-borohydride solution or by the vacuum spraying of silver.

The polyimide film metallization technology presented is different from the others described in the literature and is based on a physico-chemical approach to the formation of the metal phase that permits substantial simplification of the process and makes use of commercially available polyimide films such as Kapton $\mathrm{JP}^{\circledR}, \mathrm{HN}^{\circledast}$ (Du Pont, USA) Upilex $\mathrm{R}^{\circledR}$, $\mathrm{S}^{\circledR}$ (Ube Corp., Japan), $\mathrm{PM}^{\circledR}$ (Russia). In situ carrying out of the chemical reactions in the modified layers makes it possible to form metal phases strongly impregnated into the polyimide surfaces. The chemical metallization was implemented in three steps (I - III) with the use of different combinations of organic solvents [9].

The metalized Kapton ${ }^{\circledR}$ films have been investigated by scanning electron microscopy (SEM) using a LEO (former Cambridge) F360 microscope coupled with X-ray difractometer equipped with EDS system Oxford ISI 300.

Microindentation technique involved measuring the size of the indentation remaining on the sample surface upon removal of the loaded indenter, which is a tetrahedral diamond pyramid. Micro-hardness was determined by overall deformability of surface layers.

Multiple cracking methods included regular photographing of samples during their elongation with a digital image of sample being displayed on a monitor.

Electrical properties of samples with planar structure were investigated by direct current using silver as electrode. The electric field strength was about $20 \mathrm{mV} / \mathrm{cm}$ which avoided sample heating by electro-caloric effect within the temperature range $25{ }^{\circ} \mathrm{C}$ up to $200{ }^{\circ} \mathrm{C}$. The spectral data of reflection factors have been measured in the wavelength interval from $400 \mathrm{~nm}$ up to $750 \mathrm{~nm}$ for both facial and reverse film sides.

\section{Polyimide films were obtained in two steps:}

i interaction of the pyromellite dianhydride with oxydianiline in amide solvents to form a polyamide prepolymer,

ii the polyamide acid solution is poured gradually onto a glass substrate so that cyclisation of the amides to imides occurs within the prepolymer with the elimination of water vapour at $300^{\circ} \mathrm{C}$.

\section{RESULTS AND DISCUSSION}

The surface morphology of the two sides of the polyimide films produced by the above described procedure are different: the surface of the glass side is smooth and is marked by a high degree of luster. The air side is more dull owing to a highly developed interpenetrating system of mesopores through which water and the solvent were eliminated. Polyimide film metallization process is carried out in several steps by chemical modification : hydrolysis - conversion of the imide cycle into the salt of polyamide acid in the alkali solutions; chelating of the modified surface with metal cations and reduction of the silver incorporated in the polymer matrix.

In connection with that the process is carried out in the film so that diffusion factors along with chemical ones make a substantial contribution to and considerably complicate the process of silver coating formation. Furthermore, the different surface morphology of the two sides of the film, which is determined by polyimide film production technology, also complicates the matters. Analyzing the complicated multifactor process of heterogeneous chemical film metallization, it is necessary to separate these processes and reveal their individual effect on formation of the overall composite structure. Key factors that permit estimation of overall structure property correlations in the suggested scheme of metallization are as follows: completeness of silver reduction under conditions of the heterogeneity of the process; extent of crystallites of metal phase; character of distribution of the metal phase through the film depth; influence of the macro - and microstructures on optical properties.

Both silver-coated polyimide films and the individual components - silver and polyimide film Kapton ${ }^{\mathbb{B}}$ - were investigated. Silver coatings were obtained on quartz glass by vacuum spraying, and by chemical precipitation where silver cations were reduced on a glass substrate. Identification of $\mathrm{X}$-ray reflexes of individual components are shown in Table 1. Analysis of the data shows that chemical metallization of the polyimide films permits formation of silver coatings on high strength polyimide substrates.

The sizes of silver crystallites were determined according to the below-mentioned equation [10]:

$(B=K \lambda / \beta \cos \theta)$

The size data are depicted in Table $\mathbf{2}$. 
Table 1. Diffraction Angles (20) and Interface Distance of the Different Silver Coatings

\begin{tabular}{|c|c|c|c|c|c|c|}
\hline Sample & \multicolumn{2}{|c|}{1} & \multicolumn{2}{|c|}{2} & \multicolumn{2}{|c|}{3} \\
\hline Ag (spraying) & 38.1 & & 44.2 & & 64.25 & \\
\hline $\mathrm{Ag}$ (chem.precip.) & 38.0 & & 44.1 & & 64.25 & \\
\hline $\mathrm{Ag} / \mathrm{PI} 3$ & 38.1 & & 44.3 & & 64.7 & \\
\hline
\end{tabular}

Table 2. Silver Crystallites Sizes in the Samples of the Polyimide Films Modified in Different Ways

\begin{tabular}{|c|c|c|c|}
\hline \multirow{2}{*}{ Sample } & \multicolumn{3}{|c|}{ Crystallite size, $\mathbf{A}$} \\
\cline { 2 - 4 } & $\mathbf{1}$ & $\mathbf{2}$ & $\mathbf{3}$ \\
\hline \hline Kapton/ Ag: & 116 & 50.1 & 121 \\
-before annealing & 129 & 49.6 & 134 \\
-after annealing & 204 & 153.8 & 318.9 \\
\hline spraying Ag & & 290 & \\
\hline
\end{tabular}

It is obvious from the data presented in Table 2 that modified film 2, which used water as the hydrolysis mixture, is characterized by the smallest sized crystals. This is determined by the peculiarities of the chemical and physical interaction of water and the modifying organic solvent that results in the appearance of polymer matrix stress with ultimate formation of small size crystallites at the waterorganics interface. Films 1 and 3 contain crystallites virtually of the same size. By comparison of the silver (III) reflexes with the amorphous halo of the Kapton ${ }^{\circledR}$ film, it can be deuced that the thinnest silver coating is formed in film 3 , and the thickest one in film 1.

Considerable structural changes are observed after metalized films are annealed at $180^{\circ}$ for $10 \mathrm{~min}$. Analysis of the diffraction data shows that during annealing there occurred redistribution of reflex intensities and that for films 1 and 2 new reflexes appeared. The main factor is the strengthening of typical reflexes for all samples that testify to the degree of growth of crystallites in the silver coating. Crystallite sizes are shown in Table 2 . It can be observed that for film 1 the size of crystallites increases 1.7 times, for film 2 three times, while for film 3 the sizes ( $319 \AA$ ) exceed those in the spraycovered silver $(290 \AA)$. Sharp growth of sizes of crystallites in film 3 testifies to the ease of coalescence of silver crystallites on the surface, whereas for samples 1 and 2 the diffusion process limits formation of the larger sized crystallites due to the deeper modification of the surface.

From the analysis of diffractograms it was also seen that at the angle of $32^{\circ}$ there is a reflex (110) typical of a defective crystal lattice which ought to vanish in perfect crystals. This fact is probably determined by the substitution of the silver atom in the unit cell of the crystal lattice for one of the atoms of the polymer chain, i.e. there is formation of some chemical bonds in the coarse of annealing. This assumption can be proved by the visual adhesion that is much higher for the coatings 1 and 2 in comparison with coating 3 . The reflex at $46^{\circ}$ corresponds to formation of a new phase $-\mathrm{AgCl}$ - and is observed only in the diffractograms of the samples 1 and 2. This can be accounted for by the use of chlorinecontaining organic compounds as the modifying agent which results in the formation of silver chloride.

Microphotographs with high magnification of the silver surface (Fig. 1a) which was mechanically affected artificially (Fig. 1b) are shown in (Fig. 1). It is obvious that the coating is virtually complete. Under the main layer of the large grains there is a metal layer with grains of smaller sizes.

We studied the interaction of modified PI films with $\mathrm{AgNO}_{3}$ solutions during base-induced morphological changes. This plays a major role in the formation of metal layers on PI matrix surfaces in the presence of various solvents, such as ketone and amide type solvents, including heterocyclic ones. Absorption kinetics of $\mathrm{Ag}$ cations into a modified surface layer of PI films have been studied with potentiometer control of $\mathrm{Ag}$ ion concentration between $10^{-4}-10^{-2} \mathrm{M}$. Kapton $^{(B} 75 \& 100 \mathrm{HN}$ were used as PI films. The films were modified in alcohol-aqueous solutions and aqueous solutions of potassium alkali $\mathrm{KOH}$. Modification of films was carried out in heterocyclic, amide and ketone type solvents.

A series of kinetics curves were obtained for alicyclic films prepared at ICS and for some industrial PI films : Kapton $^{\circledR} 75 \mathrm{HN}, 100 \mathrm{HN}, 200 \mathrm{HN}$ in the $10^{-4}-10^{-2} \mathrm{M}$ concentration range of silver nitrate. The curve for Kapton ${ }^{\circledR} 100 \mathrm{HN}$ is 

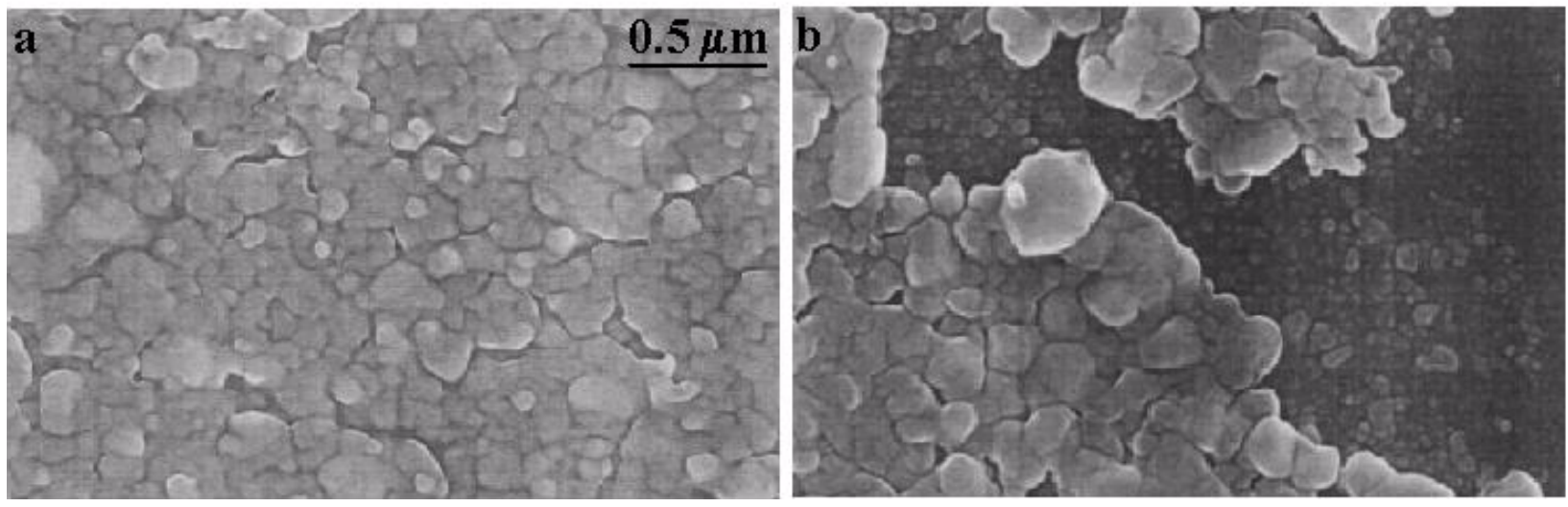

Fig. (1). SEM images of top (a) and undertow (b) layers of metalized polyimide films.

shown in Fig. (2) as an example. We can see that the curves change through a few steps:

i sharp drop of the potential at the initial step (a),

ii a gradual linear decrease (b), which corresponds to linear dependence of $\log \mathrm{C}_{\mathrm{Ag}}$ vs. time. In some curves there is a strong deviation at step (d), which corresponds to a constant concentration of $\mathrm{Ag}$ ions in the solution. Transition of the potential to the constant level relates to saturation of films by $\mathrm{Ag}$ ions.

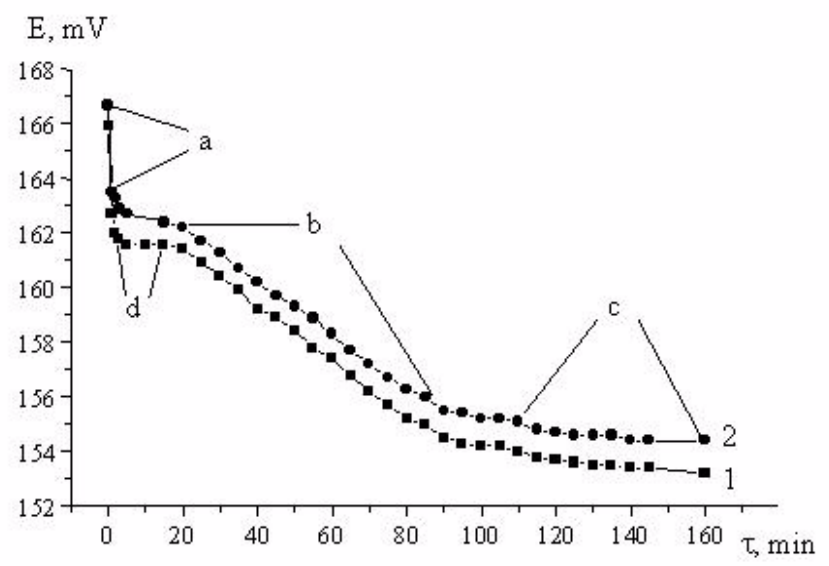

Fig. (2). Potentiometer measurement of $\mathrm{Ag}$ concentration in the aqueous $\mathrm{Ag}$ solution (the initial $\mathrm{Ag}$ concentration $10^{-4} \mathrm{M}$ ) during chelating of the modified Kapton ${ }^{(B} 100 \mathrm{HN}$ films on $1-\mathrm{Ag} / \mathrm{AgCl}$ electrode and 2- $\mathrm{Ag} / \mathrm{Ag}_{2} \mathrm{~S}$ one.

The curves vary in their dependence on concentration of chelating solution, but the slope of the curves during the linear decrease of the potential (b) remains the same for all curves. The coefficient calculated for the linear plot suggests that it involves a reduction in diffusion in the solution, but further study is required. A bending in the linear plots at the final step of the process is due to exceeding the film capacity for Ag ions, which then can not diffuse into the film. That is also confirmed by elongation of the linear plot for decreasing silver concentration. A high rate of $\mathrm{Ag}$ vanishing from the solution at the initial time, which exceeds the rate of diffusion, can be speculated as being due to dissolution of potassium polyamate. That leads to the formation of $\mathrm{Ag}$ complexes in the solution outside of the potentiometer control. Such behaviour was confirmed by measurement of the film weight. The study of the film weight before and after chelating shows a rise at $\mathrm{Ag}$ concentrations above $10^{-3} \mathrm{M}$. The decrease of weight thus manifests the dissolution of potassium salts, which are a part of the modified layers.

Complete investigation of optical properties of metalized films was carried out in the range of 400-800 $\mathrm{nm}$. Dependence of reflection coefficients of silver coatings of different sides of films 1, 2, 3 on spectrum are presented in Fig. (3). From Fig. (3) it can be seen that there are common regularities in reflection coefficients of silver coatings regardless of the way of their preparing which are identical to literature data [10] according to which the minimum of the reflection and next growth can be observed at the range of $340 \mathrm{~nm}$. At the range of $530 \mathrm{~nm}$ wherein solar radiation is the most intensive, the reflection coefficients of investigated samples are $75-90 \%$ which is much higher than literature data $[6,7]$.

Curves analysis shows that glass side of film 1 is more reflective, reflection from the air side being $5 \%$ lower. In practice, the reflectivity of the two sides is the same as is confirmed by Auger and electron microscopy data. Reflection from film 3 is worst but its sides show virtually the same value. Reflection coefficient of film 2 is $10-12 \%$ lower than film 1, but these coefficients are nevertheless higher than literature data. The difference in surface reflections of both sides of the film indicate the different morphologies of the two film surfaces determined by the method of initial polyimide film casting.

Correlation of X-ray diffraction data with the optical properties of the films show that in spite of the considerably smaller sizes of the crystallites ( $50 \AA)$ in sample 2 in comparison with samples 1 and 3 (approximately $120 \AA$ ) their reflection coefficients do not differ much. From this it follows that along with the factor of crystallite sizes in the silver coating, the morphology, or degree of "polishing", also plays a major part. In some works [5-7] the silver layer formation is carried out by high temperature thermolysis of silver in the films. Here during thermolysis the dehydrocyclisation takes place with water evolving and formation of a loose surface. In doing so, non-conductive surfaces are obtained which consist largely of carbon $(53.8 \%)$, nitrogen (3\%), oxygen (11.7\%), and fluorine (24\%) according to the analytical data. The content of the silver is $7 \%$ which indicates the existence of a silver sublayer under a layer of polymer. As shown in Fig. (1) the top layer in our films consists of $50-100-\mathrm{nm}$ size silver grains, however the first 


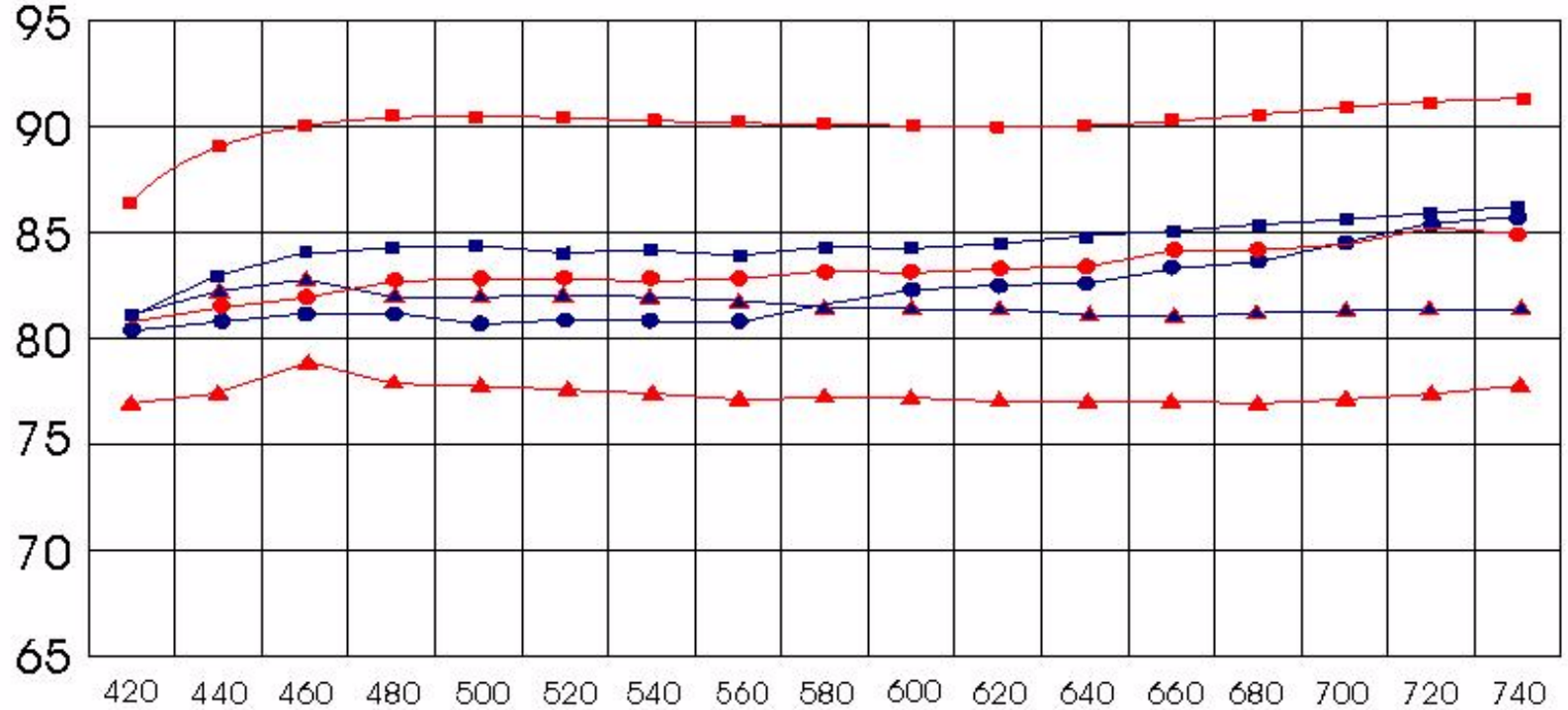

Fig. (3). Reflectivity of both glass (red) and air (blue) sides of metalized polyimide Kapton ${ }^{\circledR} 100 \mathrm{HN} / \mathrm{Ag}$ films prepared by different types of modification I- rectangle; II- round; III- triangle). X - wavelength [nm]; Y - reflectivity coefficient [\%].

sublayer consists of 8-10-nm size metal grains imbedded in a polyimide matrix.

For all presented untreated films, the depth dependence of microhardness is extreme as shown in Fig. (4). These results are determined by variation of the load value $P$ from 5 to $20 \mathrm{~g}$. For the homogenous material, the microhardness is constant or $\mathrm{H} \sim \mathrm{P} / \mathrm{D}^{2}$, where $\mathrm{D}$ is the indentation size, and the value of indenter penetration depth, $\mathrm{h}$ is proportional to D. Probably it is necessary to take into account the inhomogeneity of surface layer structure in connection with film modification. Extreme microhardness dependence with depth remains for all films after modification. Because the value of microhardness is essentially less in comparison with that of pure metal, evidently we are dealing with composite materials in which metal particles strengthen the polymer. Depth dependence of microhardness allows estimation of the depth of metal particle penetration: at this depth the two curves (for untreated films and films after modification) coincide. For Kapton ${ }^{\circledR} 100 \mathrm{NH}$ significant hardening was observed especially for cobalt - Fig. (4). The depth of metal particle penetration is about 10 micrometers. For another set of samples the depth of silver particle penetration was essentially larger.

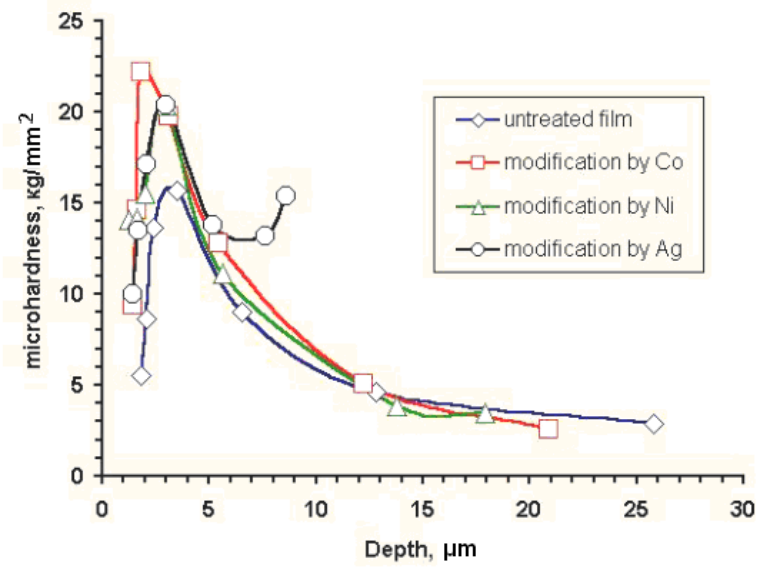

Fig. (4). Dependence of depth on microhardness for metalized Kapton ${ }^{\circledR} 100 \mathrm{HN}$ films.
Microhardness precisely correlates at SEM magnification, with layer-by-layer X-ray analysis of silver metal distribution as shown in Fig. (5).

Debonding behaviour during multiple cracking experiments with film stretching depends on the thickness of the metal coating. A thin coating does not debond from the substrate. However, if the thickness of the coating is higher than a critical value, it debonds at comparatively low strain. The critical thickness was used to determine the mode II fracture (debonding) toughness $\mathrm{G}_{\text {IIc }}$. The diagram that is typical for plastic materials is initially deformed elastically, and at strains higher than $5 \%$ the yielding of the material begins. Fracture deformation of samples was 30 to $70 \%$ depending on the substrate. The thicker was the substrate, the higher was the failure elongation. Fig. (6) shows an optical micrograph of a metalized Ag-Co surface of a 50 micron-thick Kapton $^{\circledR}$ substrate after tensile failure. A number of rather long cracks in the metal coating are observed. Obviously, this means that fracture strain of a metal coating is lower than that of a Kapton ${ }^{\circledR}$ substrate. Near the cracks a number of small bubbles are observed. Evidently, the bubbles are caused by the onset of a local debonding of the coating. Surface micro-cracks in samples based on $25 \mu \mathrm{m}$-thick Kapton ${ }^{\circledR}$ substrate were observed, while debonding of the coating film was not seen. In samples based on 8 micron-thick Kapton ${ }^{\circledR}$ films neither failure cracks nor debonding of the coating were observed.

The debonding of a coating film is characterized by a critical thickness value. A coating debonds from the substrate if its thickness exceeds the critical magnitude. By contrast, thin coatings do not debond from the substrate. For the usual method of metal sputter deposition the critical thickness of a coating for platinum/PET composite is $30 \mathrm{~nm}$. The thickness of a coating film in the $75 \mu \mathrm{m}$-thick samples was 5 to 8 microns. And only in this composite was an onset of debonding process detected. In other composites no debonding was observed at strains up to $60 \%$. This means that the adhesion of a metal film to Kapton ${ }^{\mathbb{B}}$ film is extremely high. Below the debonding of a coating is analyzed. We assume 

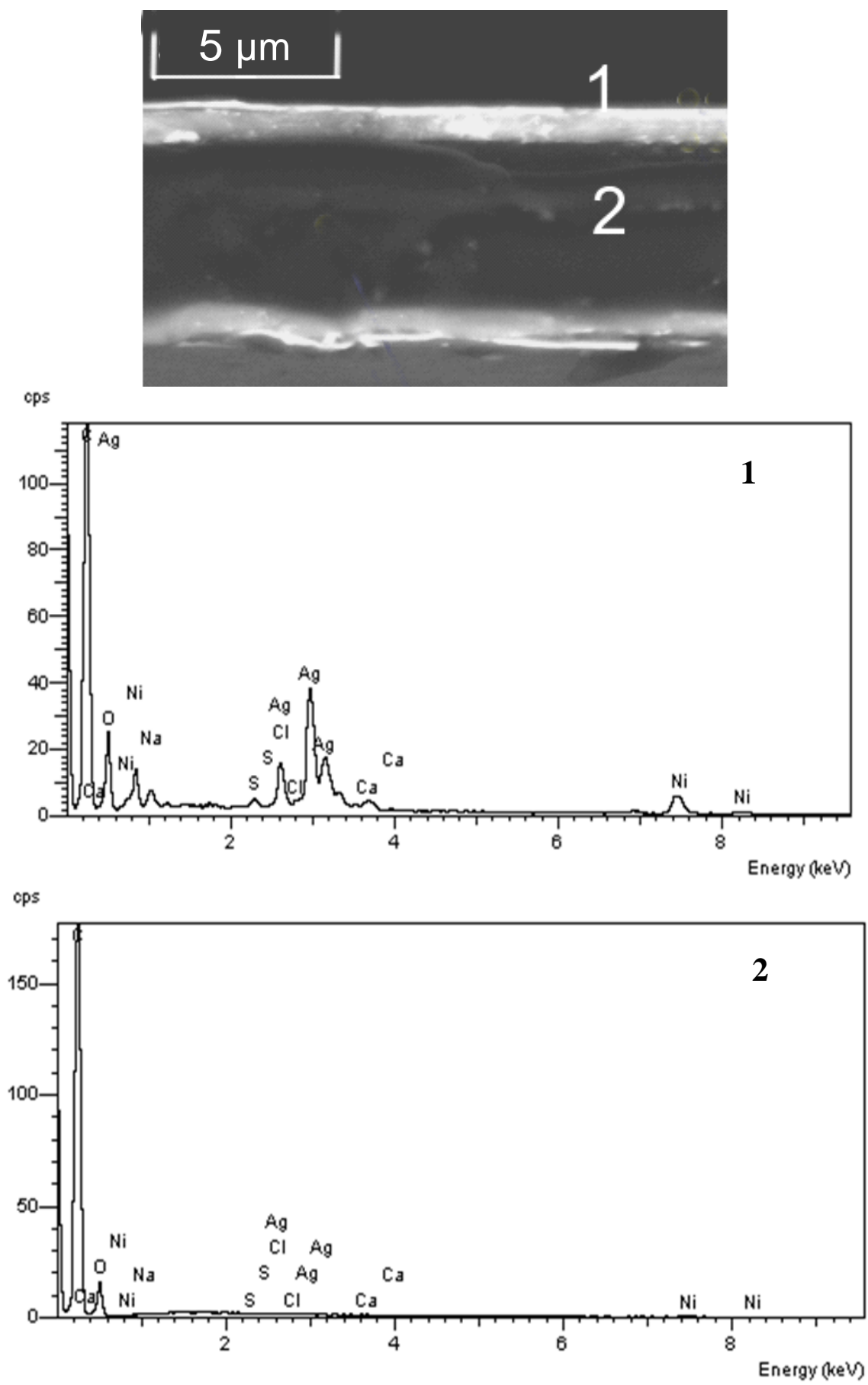

Fig. (5). Profile image of silvered Kapton ${ }^{\circledR} 100 \mathrm{HN}$ film with X-ray data for silver level distribution at the surfaces (1) and inside the film (2).

that the crack grows along the surface between the coating and the substrate. We do not know the magnitude of the stress in the coating. However, we can roughly estimate it. The lower estimation of the stress is $150 \mathrm{MPa}$, the yield stress of the substrate. The yielded stresses of $\mathrm{Ag}$ and $\mathrm{Ni}$ are 140-180 and $400 \mathrm{MPa}$ respectively. The Young's modulus of $\mathrm{Ag}$ is $70 \mathrm{GPa}$. Substituting $\sigma=160 \mathrm{MPa}, \mathrm{E}=70 \mathrm{GPa}$ and $\mathrm{h}^{*}=7 \mu \mathrm{m}$, we get $\mathrm{G} \approx 1.3 \mathrm{~J} / \mathrm{m}^{2}$. This value is approximately hundreds-fold higher than the debonding value for metal films made by the usual sputter deposition method.
The preparation of metalized polyimide films by in situ chemical modification provides for high electroconductivity and reflectivity. The construct could be an ideal candidate for the substrate in flexible mirrors and microchips.

\section{CONCLUSION}

The employment of in situ chemical reactions in the modified near-surface layers of PI films allows us to impregnate metals strongly into the films avoiding completely poor metal-PI adhesion. Such an inexpensive, technically uncomplicated procedure for metallization of PI films allows 


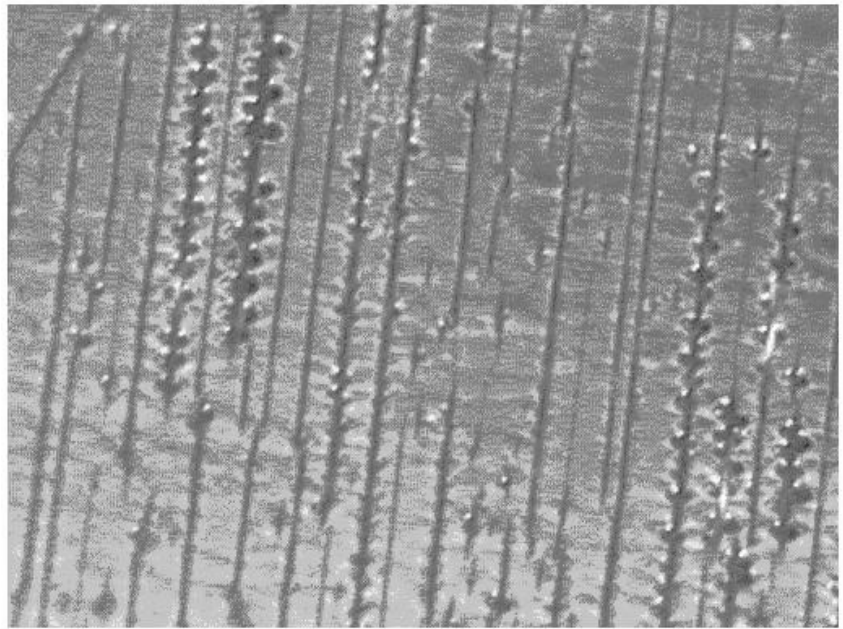

Fig. (6). Optical micrograph of Ag-Co metalized surface of a 50 micron-thick Kapton ${ }^{\circledR}$ substrate after tensile failure.

production of reflective and electro-conductive metalized PI constructs with fine integral optical characteristics (flexible mirrors).

Measurements of reflectivity in the visible range and surface resistance at elevated temperatures show that reflectivity coefficients of silvered films achieve $90-92 \%$ and surface resistance is about $0.5 \mathrm{ohm} / \mathrm{cm}$.

Deposition of a second metal coating by electrochemical process on the silvered polyimide films improves the stability and the reflectivity up to $98 \%$. Electroconductive silver layer provides the possibility for deposition of different metals: palladium, platinum, gold, rhodium, cobalt, nickel and their alloys in mild conditions. This treatment increases the stability to the effect of atomic oxygen (main damaging factor of space environment), raising abrasive stability and giving no delaminating effect. Thermal stability of metalized films is presented and discussed.

In view to broaden thermo-mechanical behaviour of PI materials itself and to regulate optical and physical properties of their metalized constructs, new polymeric blends based on alicyclic polyimide and polycarbonate or polysulfone have been prepared and studied [2].

The use of such metalized reflectors as solar energy concentrators with high electroconductivity to dissipate charges can remarkably reduce the weight and fragility of mirrors, providing good flexibility of packing for subsequent deployment in space applications. Further development of metallization technology will lead to their use in microelectronics as printed circuits, chips and surface-conductive microboards.

\section{ACKNOWLEDGEMENT}

This research is supported by NATO in the framework science programme of Science for Peace, project NATO SfP \# 978013 "Creation of New Strong Metal / Polyimide Films with Definable Reflectivity \& Electroconductivity”.

\section{REFERENCES}

[1] S.K. Kudaikulova, O. Prikhodko, G. Boiko, B.A. Zhubanov, V. Yu. Voytekunas and M.J.M. Abadie, "Metalized Polyimide Films with High Relectivity and Electroconductivity", Polyimides and Other High Temperature Polymers, Volume 2, Editor : K.L. Mittal, VSP Utrecht. Boston, pp. 389-406, 2003.

[2] S. K. Kudaikulova, O. Prikhodko, G. Boiko, B.A. Zhubanov, A Kurbatov, T. Akmetov, V. Yu. Voytekunas and M.J.M. Abadie, Eurasian Chemico-Technological J., vol. 6 (1), 1-66, 2004.

[3] E.L. Vecherkina, S.K. Kudaikulova, R.M. Iskakov, B.A. Zhubanov, V. Yu. Voytekunas and M.J.M. Abadie, "Metalized Polyimide Films: Metallization and Mechanism of the Process", Polymer Science, vol. 49A(2), pp.142-147, 2007.

[4] D.M. Stoakley and A.K.S. Clair, "Synthesis and Characterization of Self-metalizing Palladium-doped Polyimide Films", Polym. Prepr., vol. 37(1), 541-548, 1996.

[5] R.E. Southward, D.S. Thompson and A.K.S. Clair, "Control of Reflectivity in Metalized Polyimide Films Prepared via in Situ Silver (I) Reduction", Chem. Mater., vol. 2(8), 501-510, 1997.

[6] R.E. Southward, C.M. Bogges, D.W. Thompson and A.K.S. Clair, "Synthesis of Surface Metalized Polyimide Films via in Situ Reduction of Perfluoroalkanoate Silver (I) Complexes in Polyamic Acid Precursors", Chem. Mater., vol. 10(2), 1408-1415, 1998.

[7] R.E. Southward, D.S. Thompson, D.W. Thompson and A.K.S Clair, "Inverse Chemical Deposition : A Novel Single Stage Synthesis of Highly Reflective and Conductive Silvered Polymeric Films", Chem. Mater., vol. 11(2), 501-508, 1999.

[8] J. Rosolovsky, R.K. Bogges, A.F. Rubira, L.T. Taylor, D.M. Stoakle and A.K.S. Clair, "Super Critical Fluid Infusion of Silver into Polyimide Films of Varying Chemical Composition", Polym. Prepr., vol. 38(1), 282-290, 1997.

[9] S.K. Kudaikulova, V.L. Lipik, V. Yu. Voytekunas, A. Périchaud and M.J.M. Abadie, "Métallisation de Films Polyimide. Mise au Point du Traitement Chimique", Revue Roumaine de Chimie, vol. 52 (4), In press 2007.

[10] Y.A. Masterov and Y.V. Saksonov, "Silver, Alloys and Bimetals on its Basis", Ed. Moscow: Academic Press RAN, pp. 296-309 (in Russian) 2005. 